\title{
Exteroceptive reflex myoclonus: clinical and electrophysiological study
}

\author{
M GONCE, ${ }^{*} \dagger$ PJ DELWAIDE* \\ From Service de Neurologie-Neurophysiologie Clinique, Hôpital Universitaire de Bavière, ${ }^{*}$ and Service de \\ Neurochirurgie-Neurologie; Clinique St Joseph, $\dagger$ Liège, Belgium
}

SUMmaRY Clinical and electrophysiological data are reported on two patients presenting with unusual reflex myoclonus triggered specifically by exteroceptive stimulation.

Myoclonus may occur spontaneously or may be triggered by various stimuli ("reflex myoclonus"). Cortical and reticular reflex myoclonus, both of which are electrophysiologically ${ }^{1}$ considered to depend on overactivity in long loop pathways, have been distinguished. In almost all the reported cases, reflex myoclonus was triggered by proprioceptive afferent discharges; in a few patients, exteroceptive cutaneous stimuli were responsible. ${ }^{2-4}$

We report clinical and neurophysiological data on two more cases of pure exteroceptive reflex myoclonus.

\section{Electrophysiological techniques}

EEG, EMG cutaneous recordings of spontaneous or provoked myoclonus, somatosensory evoked potentials were recorded using conventional techniques. ${ }^{5} 6$ Jerk locked averaging EEG was performed following the method described by Shibasaki et al. ${ }^{7}$

\section{Case reports}

Patient I This 56-year-old man with known coronary artery disease was admitted to the intensive care unit after a myocardial infarction. Within 3 days, he had two cardiac arrests each of 5-10 minutes duration and recovered. Five days later myoclonic jerks were observed with increasing intensity. They were present at rest but were specifically triggered by light cutaneous stimuli applied to the skin overlying the four limbs and particularly the fingers. Voluntary movements, tendon taps, noise, passive displacement of joints were not really effective in triggering myoclonus. The patient

Address for reprint requests: Dr PJ Delwaide, Hôpital Universitaire de Bavière, Boulevard de la Constitution 66, 4020 Liège, Belgium.

Received 8 November 1985 and in revised form 29 January 1986. Accepted 3 February 1986 was conscious, well oriented and had no upper motoneuron signs. Successive treatment with clonazepam, phenobarbital and valproic acid, was ineffective. The patient died three weeks later from a chest infection. Necropsy was not performed. Electrophysiological studies were carried out serially during his time in hospital. Routine EEG showed a generalised slowing with sporadic pseudo-rhythmical reinforcements. Spikes and multispikes were recorded diffusely. Spontaneous myoclonic jerks were recorded as di- or polyphasic potentials of brief duration $(10$ to $50 \mathrm{~ms})$. They were arhythmic and mostly synchronous in the extensor and flexor muscles of the fingers. Some jerks were preceded by a widespread cortical spike-discharge, but involving particularly the contralateral motor area (delay 20 to $25 \mathrm{~ms}$ for upper limbs).

Myoclonus could be evoked by non nociceptive stimulation of interdigital nerves of the hand. Thus, stimulation of the index finger constantly evoked a late response in the ipsilateral abductor pollicis brevis (APB) at a mean latency of $60.8 \mathrm{~ms}$ (range 54 to 65 ). In 50 to $60 \%$ of instances, a second, third and even fourth response could be recorded (see fig, C). These responses had the same appearance and duration as the spontaneous myoclonus (fig, A). They were also evoked at a considerable distance from the stimulation point, for example in the contralateral abductor pollicis brevis and the ipsilateral quadriceps. Sural nerve stimulation at the ankle evoked similar late events, for example in the ipsilateral tibialis anterior at a mean delay of $117 \mathrm{~ms}$ (113 to $125 \mathrm{~ms}$ ). On the contrary, slow and fast passive finger displacement to stretch the finger extensor and flexor muscles, did not elicit any reflex myoclonic jerks. Stimulations applied on the median nerve at the wrist evoked less regularly one or two late responses in the ipsilateral APB or in contralateral upper and lower limb muscles at shorter delays than index stimulation, for example $54.7 \mathrm{~ms}(51$ to $57 \mathrm{~ms})$ in ipsilateral APB. A third or fourth late response was never obtained with this procedure. Somesthetic evoked potentials appeared at normal latencies but with high amplitude: $11 \mu \mathrm{V}$ (peak to peak from N20 to P25) after median nerve stimulation (fig, B), $7 \cdot 5 \mu \mathrm{V}$ after sural nerve stimulation.

Patient 2 This 52-year-old woman presented with a 1 year 

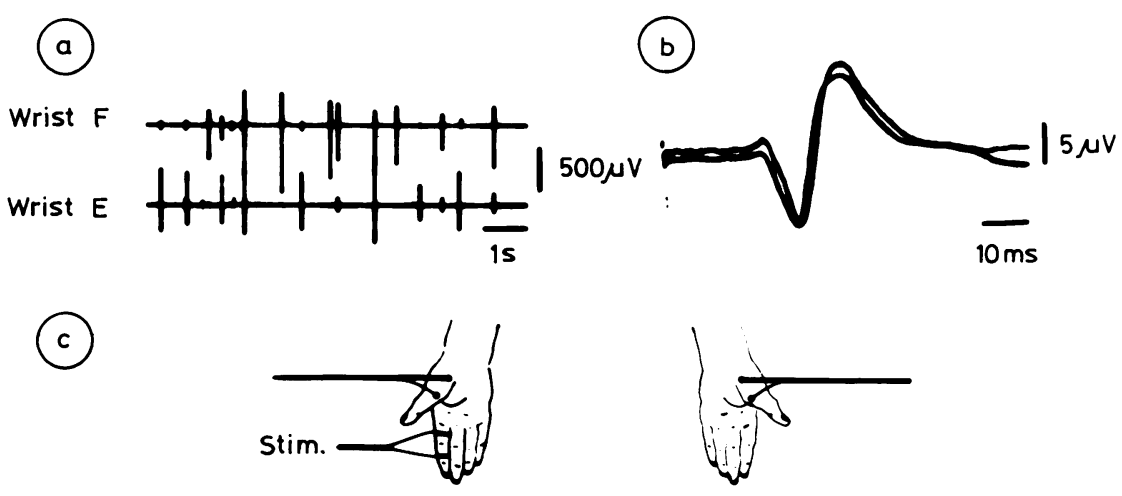

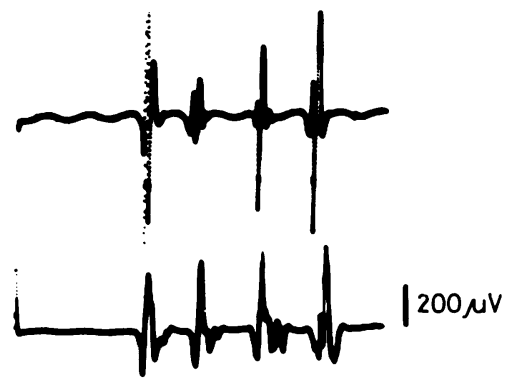

$20 \mathrm{~ms}$

Right Abd. Pol. Brevis
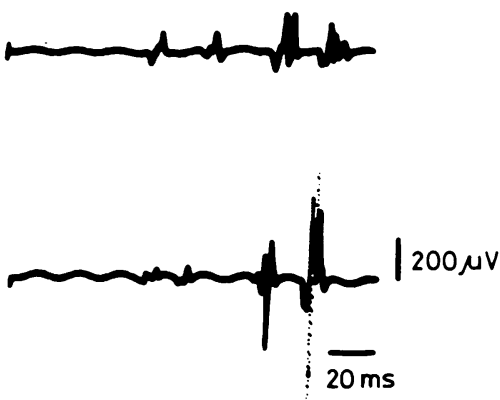

Left Abd. Pol. Brevis

Fig (A) Spontaneous discharges recorded in wrist flexor and extensor respectively. (B) Giant somatosensory evoked potentials following index finger stimulation (superimposed curves of 128 sweeps each). (C) Scheme of the experimental set up. The right index finger is stimulated (single shocks, 1 ms duration). Recordings are made from both abductor pollicis brevis muscles. Repetitive discharges are regularly observed not only on the right side but also on the left.

history of dementia and abnormal behaviour. At admission, stiffness and myoclonic jerks were observed. They occurred spontaneously or could be consistently elicited by light touch of skin. The sensitive area of skin was widespread both in upper and lower limbs and the myoclonic jerks were maximum in the vicinity of the touched place. CT scan showed slight cortical atrophy and enlarged ventricles. RISA and spinal fluid examinations were normal. No toxic cause for the myoclonus could be detected. Treatment with clonazepam had no effect. Progabide, a GABA-mimetic agent $(300 \mathrm{mg}$ tid) caused a remarkable reduction of myoclonus which disappeared 5 days after the onset of treatment. A fortnight later, progressive withdrawal of progabide was not followed by return of the myoclonic jerks. At the present time, the patient is severely demented and resident in a psychogeriatric unit.

EEG showed diffuse theta and delta waves. At times, bursts of pseudo-rhythmical high amplitude multispikes were recorded in the frontal regions. Spontaneous myoclonic jerks were rare, asynchronous in the extensors and flexors of the fingers. They were brief: 10 to $35 \mathrm{~ms}$. Using the jerk-locked back averaging procedure, no EEG abnormal event was time locked with the myoclonus.

Stimulation of index interdigital nerves regularly evoked repetitive reflex myoclonic jerks in the ipsilateral APB (latencies: from 44 to $64 \mathrm{~ms}, 66$ to $98 \mathrm{~ms}$ and 114 to 140 for the first, second and third responses respectively). This stimulation also evoked regularly late responses in non-distant muscles such as the ipsilateral long supinator. However, the first late response was rare in distant muscles, for example less than $20 \%$ in quadriceps, contralateral long supinator or cephalic muscles. At cephalic level, the responses appeared in a fixed order, first in the masseter, second in orbicularis oris and third in sternocleidomastoid muscles. Passive stretch of forearm muscles did not induce reflex myoclonus. Median nerve stimulation at the wrist evoked similar responses but more irregularly. Latencies and amplitudes of somatosensory evoked potentials were within normal limits. After 3 days of progabide, reflex myoclonus became rarer and reduced in amplitude. 


\section{Discussion}

The patients described in this report are examples of reflex myoclonus triggered by exteroceptive stimulation.

The pure exteroceptive origin of a myoclonic jerk may be difficult to prove clinically. Even in the absence of overt motility, proprioceptive information may be postulated (for example from spindles and Golgi organs), which might play a role in myoclonus suspected to be of exteroceptive origin. First the spindles are excessively sensitive to very small displacements and could be activated by just touching the skin. Furthermore even if a patient appears to be motionless, discrete contractions cannot be ruled out. Electrical stimulation of interdigital nerves can counter these objections even if a small contribution of proprioceptive joint and tendinous afferents cannot definitely be excluded. Our electrophysiological data show that stimulation of purely sensory nerves is more efficient in eliciting myoclonus than mixed nerve stimulation.

Sutton and Mayer ${ }^{2}$ reported the case of a patient with myoclonus, restricted to the right side of the body, due to a large atrophic vascular lesion involving the left frontotemporoparietal region. Stimulation of index finger or median nerve at wrist elicited a very large somatosensory evoked potential (SEP) and a myoclonic jerk. They termed this a "C-reflex" assuming that it was reflecting cortical reflex activity. Its mean latency was $51 \mathrm{~ms}$ (range $48-54 \mathrm{~ms}$ ) and sometimes a second component could be recorded 35 to $50 \mathrm{~ms}$ later. One year later, Sutton ${ }^{8}$ demonstrated that the critical triggering stimulus was touch or skin pressure and not movement or muscular stretching. Our results are in close agreement with those reported by Sutton and Mayer. ${ }^{2}$ Latencies of these cases of reflex myoclonus are compatible with an exteroceptive long loop pathway relaying in the brain. Existence of such long loops in humans has been shown by Jenner and Stephens ${ }^{9}$ and Delwaide and Crenna. ${ }^{10}$ In pathological situations the gain of these long loops could be increased and easily explain exteroceptive reflex myoclonus. Marsden et $a^{1}$ proposed an electrophysiological classification of myoclonus, based originally on their work in patients with post-hypoxic action myoclonus. They proposed spontaneous or reflex cortical and subcortical types. In cortical myoclonus the jerk is generated by a discharge arising in the cerebral cortex which has become oversensitive, for example to peripheral stimulation, leading to cortical reflex myoclonus. In contrast, reticular myoclonus is supposed to originate within the brainstem itself (perhaps from discharges in the nucleus gigantocellularis medullaris, ${ }^{11}$ the abnormal activity being propagated up to the brain and down to the periphery to produce muscle jerks. According to this electrophysiological classification, our first reported case could represent an exteroceptive form of cortical reflex myoclonus. However it is unusual because it is not focal with respect to proprioceptive reflexes but generalised. In this way, it is not similar to the case No 1 of Obeso et al ${ }^{4}$ of pure cutaneous reflex myoclonus in which the stimulussensitive fields were restricted to plantar and palmar skin areas; in the same way, the myoclonic jerks were multifocal. It is possible that a cortical discharge could trigger a brainstem reticular discharge and finally cause generalisation of myoclonus responses, a mechanism already suspected by Marsden et $a l^{1}$ and called "cortically triggered reticular myoclonus". Our observation also differs from the cases of pure or combined cutaneous reflex myoclonus described by Obeso $\mathrm{et} \mathrm{al}^{4}$ in which the size of the N1 component of the SEP, which represents the arrival of the afferent volley in the sensory cortex, was normal. This component is enlarged in our case suggesting an abnormal sensory input into cortex as in the case of Jones. ${ }^{12}$ The second case is more difficult to classify and does not fit strictly the criteria either of cortical myoclonus or reticular myoclonus. There is no jerk-locked back averaged wave but this lack could be explained by the fact that spontaneous jerks were rare and asynchronous. This condition could represent an insurmountable problem to the technique of backaveraging. Afferent input from one jerk may interfere with any potentials preceding jerks in a different muscle. Two arguments could be put forward in favour of a cortical origin: myoclonus is multifocal and progress in a descending order of activation in cranial nerve nuclei. If true our second case would resemble myoclonus of Shibasaki's type II $^{13}$ which is believed to reflect a hyperexcitability of the efferent system.

Pure exteroceptive myoclonus has so far been reported in an atrophic vascular lesion, ${ }^{2}$ in a patient after cardiac arrest ${ }^{3}$ and in sporadic olivopontocerebellar atrophy. ${ }^{4}$ In this report an additional case of anoxic encephalopathy (patient No 1) and a patient with dementia of obscure origin (patient No 2) with a similar disturbance were described.

\section{References}

${ }^{1}$ Marsden CD, Hallett M, Fahn S. The nosology and pathophysiology of myoclonus. In: Marsden CD, Fahn S, eds. Movement Disorders. London: Butterworth 1981.

${ }^{2}$ Sutton GG, Mayer FS. Focal reflex myoclonus. $J$ Neurol Neurosurg Psychiatry 1974;37:207-17.

${ }^{3}$ Niedermeyer E, Bauer G, Burnite R, Reichenbach D. Selective stimulus-sensitive myoclonus in acute cerebral anoxia. Arch Neurol 1977;34:365-8.

${ }^{4}$ Obeso JA, Rothwell JC, Marsden CD. The spectrum of 
cortical myoclonus. Brain 1985;108:193-224.

${ }^{5}$ Hallett M, Chadwick D, Adam I, Marsden CD. Reticular reflex myoclonus: a physiological type of human posthypoxic myoclonus. J Neurol Neurosurg Psychiatry 1977;40:253-64.

${ }^{6}$ Hallett M, Chadwick D, Marsden CD. Cortical reflex myoclonus. Neurology 1979;29:1107-25.

${ }^{7}$ Shibasaki H, Yamashita Y, Kuroiwa Y. Electroencephalographic studies of myoclonus. Myoclonus related cortical spikes and high amplitude somatosensory evoked potentials. Brain 1978;101:447-60.

${ }^{8}$ Sutton GG. Receptors in focal reflex myoclonus. $J$ Neurol Neurosurg Psychiatry 1975;38:505-7.

${ }^{9}$ Jenner IR, Stephens IA. Cutaneous reflex responses and their central pathways studied in man. J Physiol (Lond)
1982;333:405-19.

${ }^{10}$ Delwaide PJ, Crenna P. Cutaneous nerve stimulation and motoneuronal excitability. II: Evidence for non segmental influences. J Neurol Neurosurg Psychiatry 1981; 44:699-707.

${ }^{11}$ Zuckerman EG, Glaser GH. Urea-induced myoclonic seizures. Arch Neurol 1982;27:14-28.

12 Jones SJ. Somatosensory evoked potentials: the abnormal waveform. In: Halliday AM, ed. Evoked Potentials in Clinical Testing. London: Churchill Livingstone 1982: 462-5.

${ }^{13}$ Shibasaki H, Yamashita Y, Neshige R, Tobimatsu S, Fukui R. Pathogenesis of giant somatosensory evoked potentials in progressive myoclonic epilepsy. Brain 1985;108:225-40. 